\title{
LINEAR MAPS PRESERVING THE IDEMPOTENCY OF JORDAN PRODUCTS OF OPERATORS*
}

\author{
LI FANG ${ }^{\dagger}$
}

\begin{abstract}
Let $\mathcal{B}(\mathcal{X})$ be the algebra of all bounded linear operators on a complex Banach space $\mathcal{X}$ and let $\mathcal{I}^{*}(\mathcal{X})$ be the set of non-zero idempotent operators in $\mathcal{B}(\mathcal{X})$. A surjective map $\varphi: \mathcal{B}(\mathcal{X}) \rightarrow \mathcal{B}(\mathcal{X})$ preserves nonzero idempotency of the Jordan products of two operators if for every pair $A, B \in \mathcal{B}(\mathcal{X})$, the relation $A B+B A \in \mathcal{I}^{*}(\mathcal{X})$ implies $\varphi(A) \varphi(B)+\varphi(B) \varphi(A) \in \mathcal{I}^{*}(\mathcal{X})$. In this paper, the structures of linear surjective maps on $\mathcal{B}(\mathcal{X})$ preserving the nonzero idempotency of Jordan products of two operators are given.
\end{abstract}

Key words. Banach space, Preserver, Idempotent, Jordan product.

AMS subject classifications. 47B49.

1. Introduction. This paper is a continuation of our recent work on preserver problems concerning certain properties of products or triple Jordan products of operators [3, 4, 13], and the related works in [2] and [7].

Let $\mathcal{X}$ be a complex Banach space, and let $\mathcal{B}(\mathcal{X})$ be the algebra of all bounded linear operators on $\mathcal{X}$. The dual of $\mathcal{X}$ is denoted by $\mathcal{X}^{\prime}$ and the adjoint of $T \in \mathcal{B}(\mathcal{X})$ by $T^{\prime}$. Let $\mathcal{I}^{*}(\mathcal{X}), \mathcal{I}_{1}(\mathcal{X})$ and $\mathcal{N}_{1}(\mathcal{X})$ be the set of nonzero idempotent operators, the set of rank-one idempotent operators and the set of rank-one nilpotent operators in $\mathcal{B}(\mathcal{X})$, respectively. If $\mathcal{X}$ has dimension $n$ with $2 \leq n<\infty$, then $\mathcal{B}(\mathcal{X})$ is identified with the algebra $\mathcal{M}_{n}$ of $n \times n$ complex matrices and $\mathcal{I}_{n}(\mathcal{X})$ refers to the set of idempotent matrices in $\mathcal{M}_{n}$. For an operator $T \in \mathcal{B}(\mathcal{X})$, the range, the kernel and the rank of $T$ are denoted by $R(T), N(T)$ and $\operatorname{rank} T$, respectively. Let $\mathcal{F}(\mathcal{X})$ and $\mathcal{F}_{1}(\mathcal{X})$ denote the set of finite rank operators and the set of rank-one operators in $\mathcal{B}(\mathcal{X})$, respectively. For a non-zero vector $x \in \mathcal{X}$ and a non-zero $f \in \mathcal{X}^{\prime}$, we denote by $x \otimes f$ the rank one operator defined by $(x \otimes f) y=f(y) x, y \in \mathcal{X}$. Note that every bounded linear rank one operator on $\mathcal{X}$ can be written in this form. The rank-one operator $x \otimes f$ is an idempotent operator if and only if $f(x)=1$, and $x \otimes f$ is a nilpotent operator if and only if $f(x)=0$. Given $P, Q \in \mathcal{I}(\mathcal{X}), P \leq Q$ if $P Q=Q P=P$ and $P<Q$ if $P \leq Q$ with $P \neq Q$.

${ }^{*}$ Received by the editors on November 7, 2010. Accepted for publication on July 2, 2011. Handling Editor: Bit-Shun Tam.

${ }^{\dagger}$ Department of Mathematics, Northwest University, Xi'an 710127, China (fangli@nwu.edu.cn). Supported by the National Natural Science Foundation of China (No. 11026134) and the Special Research Project of Educational Department of Shaanxi Province (No. 09JK741). 
In this paper, we are interested in determining the structure of linear surjective maps $\varphi: \mathcal{B}(\mathcal{X}) \rightarrow \mathcal{B}(\mathcal{X})$ for every pair $A, B \in \mathcal{B}(\mathcal{X})$ having the property that

$$
A B+B A \in \mathcal{I}^{*}(\mathcal{X}) \Rightarrow \varphi(A) \varphi(B)+\varphi(B) \varphi(A) \in \mathcal{I}^{*}(\mathcal{X}) .
$$

We will derive the following two theorems regarding the structure.

ThEOREM 1.1. Let $\varphi$ be a linear map on $\mathcal{M}_{n}$ with $n \geq 3$. Then $\varphi$ preserves the nonzero idempotency of Jordan products of two operators if and only if there exist an invertible matrix $A \in \mathcal{M}_{n}$ and a constant $\lambda \in\{1,-1\}$ such that one of the following holds.

(1) $\varphi(X)=\lambda A X A^{-1}$ for all $X \in \mathcal{M}_{n}$;

(2) $\varphi(X)=\lambda A X^{t} A^{-1}$ for all $X \in \mathcal{M}_{n}$, where $X^{t}$ is the transpose of $X$.

TheOREM 1.2. Let $\mathcal{X}$ be a complex infinite dimensional Banach space and let $\varphi$ be a linear surjective map on $\mathcal{B}(\mathcal{X})$. Then $\varphi$ preserves the nonzero idempotency of Jordan products of two operators if and only if there exist a bounded invertible linear or conjugate-linear operator $A: \mathcal{X} \rightarrow \mathcal{X}$ and a constant $\lambda \in\{1,-1\}$ such that

$$
\varphi(X)=\lambda A X A^{-1} \text { for all } X \in \mathcal{B}(\mathcal{X}),
$$

or, only if $\mathcal{X}$ is reflexive, there exist a bounded invertible linear or conjugate-linear operator $A: \mathcal{X}^{\prime} \rightarrow \mathcal{X}$ and a constant $\lambda \in\{1,-1\}$ such that

$$
\varphi(X)=\lambda A X^{\prime} A^{-1} \text { for all } X \in \mathcal{B}(\mathcal{X})
$$

2. Preliminary results. Assume that $\mathcal{X}$ is a complex Banach space with dimension at least 3 . In this section, we introduce some elementary results that will be used in the proofs of main theorems.

Definition 2.1. [11] Let $\mathcal{U}$ and $\mathcal{V}$ be vector spaces over a filed $\mathbb{F}$. Linear operators $T_{1}, \ldots, T_{n}: \mathcal{U} \rightarrow \mathcal{V}$ are locally linearly dependent if $T_{1} u, \ldots, T_{n} u$ are linearly dependent for every $u \in \mathcal{U}$.

Lemma 2.2. [11] Assume that the operators A, B, I are locally linearly dependent. Then there exist scalars $\lambda$ and $\mu$ such that $(A-\lambda)(B-\mu)=0$ and either $(A-\lambda)^{2}=0$ or $(B-\mu)^{2}=0$.

Lemma 2.3. Let $P, Q \in \mathcal{B}(\mathcal{X})$ be two idempotent operators. Then $z P+(1-z) Q \in$ $\mathcal{I}^{*}(\mathcal{X})$ for any $z \in \mathbf{C} \backslash\{0,1\}$ if and only if $P+Q=P Q+Q P$.

Proof. We obtain the desired result by direct calculation. $\square$ 
Lemma 2.4. Let $A \in \mathcal{B}(\mathcal{X})$ and $x \otimes f \in \mathcal{B}(\mathcal{X})$ be with $f(x) \neq 0$. Then $A x \otimes f+$ $x \otimes f A \in \mathcal{I}^{*}(\mathcal{X})$ if and only if $A x=\frac{1}{2 f(x)} x$ or $A^{\prime} f=\frac{1}{2 f(x)} f$.

Proof. Note that $A x \otimes f+x \otimes f A \in \mathcal{I}^{*}(\mathcal{X})$ implies that $A x \otimes\left[f(A x) f+f(x) A^{\prime} f-\right.$ $f]+x \otimes\left[f\left(A^{2} x\right) f+f(A x) A^{\prime} f-A^{\prime} f\right]=0$. If $A x$ and $x$ as well as $A^{\prime} f$ and $f$ are linearly independent, then $f(A x)=1, f(x)=0$ and $f\left(A^{2} x\right)=0$. But this contradicts the hypothesis $f(x) \neq 0$. Hence, either $A x$ and $x$ are linearly dependent or $A^{\prime} f$ and $f$ are linearly dependent. Then we can compute that $A x=\frac{1}{2 f(x)} x$ or $A^{\prime} f=\frac{1}{2 f(x)} f$.

The idea of the following lemma comes from [10].

Lemma 2.5. Let $A \in \mathcal{B}(\mathcal{X})$ be any non-scalar operator and let $\alpha$ be any fixed complex number. Then there exists an idempotent operator $P \in \mathcal{B}(\mathcal{X})$ of rank one such that $\alpha$ is an eigenvalue of $A+2 P$.

Proof. As $A$ is a non-scalar operator, we can find $x \in \mathcal{X}$ such that $x$ and $A x$ are linearly independent. Define $P \in \mathcal{B}(\mathcal{X})$ by

$$
P x=\frac{\alpha}{2} x-\frac{1}{2} A x, \quad P A x=\alpha\left(\frac{\alpha}{2}-1\right) x-\left(1-\frac{\alpha}{2}\right) A x \quad \text { and } \quad P z=0
$$

for every $z \in \mathcal{X} \ominus[x, A x]$. Clearly, $P$ is an idempotent operator of rank one and $(A+2 P) x=\alpha x$.

3. Main results. Assume that $\mathcal{X}$ is a complex Banach space with dimension at least 3 and we consider a linear surjective map $\varphi: \mathcal{B}(\mathcal{X}) \rightarrow \mathcal{B}(\mathcal{X})$ preserving the nonzero idempotency of Jordan products of operators, that is, $\varphi(A) \varphi(B)+$ $\varphi(B) \varphi(A) \in \mathcal{I}^{*}(\mathcal{X})$ whenever $A B+B A \in \mathcal{I}^{*}(\mathcal{X})$ for every pair $A, B \in \mathcal{B}(\mathcal{X})$.

LEMMA 3.1. Let $\varphi$ be as above. Then $\varphi$ is injective.

Proof. Assume that $\varphi(A)=0$ for some non-zero operator $A \in \mathcal{B}(\mathcal{X})$. Suppose that there exists an $x \in \mathcal{X}$ such that $x, A x$ and $A^{2} x$ are linearly independent. Then there is an $f \in \mathcal{X}^{\prime}$ such that $f(x)=f\left(A^{2} x\right)=0$ and $f(A x)=1$. It follows that $A x \otimes f+x \otimes f A \in \mathcal{I}^{*}(\mathcal{X})$. But, $\varphi(A) \varphi(x \otimes f)+\varphi(x \otimes f) \varphi(A)=0 \notin \mathcal{I}^{*}(\mathcal{X})$. This contradiction implies that $x, A x$ and $A^{2} x$ are linearly dependent for every $x \in \mathcal{X}$. By Lemma 2.2, there exist scalars $\lambda$ and $\mu$ such that $(A-\lambda)\left(A^{2}-\mu\right)=0$ and either $(A-\lambda)^{2}=0$ or $\left(A^{2}-\mu\right)^{2}=0$.

If $(A-\lambda)\left(A^{2}-\mu\right)=0$ and $(A-\lambda)^{2}=0$, then $\left(\mu-\lambda^{2}\right) A=\lambda\left(\mu-\lambda^{2}\right)$. When $\mu-\lambda^{2} \neq 0$, we get that $A=\lambda I$. For any pair $x_{1} \in \mathcal{X}$ and $f_{1} \in \mathcal{X}^{\prime}$ with $f_{1}\left(x_{1}\right)=\frac{1}{2 \lambda}$, we have $A x_{1} \otimes f_{1}+x_{1} \otimes f_{1} A \in \mathcal{I}^{*}(\mathcal{X})$ and then $\varphi(A) \varphi\left(x_{1} \otimes f_{1}\right)+\varphi\left(x_{1} \otimes f_{1}\right) \varphi(A) \in \mathcal{I}^{*}(\mathcal{X})$. However, this contradicts $\varphi(A) \varphi\left(x_{1} \otimes f_{1}\right)+\varphi\left(x_{1} \otimes f_{1}\right) \varphi(A)=0$. When $\mu-\lambda^{2}=0$ and $\lambda \neq 0$, we know that $A-\lambda I$ is a nilpotent operator and there is a non-zero vector $x_{2} \in \mathcal{X}$ such that $A x_{2}=\lambda x_{2}$. Selecting $f_{2} \in \mathcal{X}^{\prime}$ with $f_{2}\left(x_{2}\right)=\frac{1}{2 \lambda}$, we get $A x_{2} \otimes f_{2}+x_{2} \otimes f_{2} A \in \mathcal{I}^{*}(\mathcal{X})$, which implies $\varphi(A) \varphi\left(x_{2} \otimes f_{2}\right)+\varphi\left(x_{2} \otimes f_{2}\right) \varphi(A) \in \mathcal{I}^{*}(\mathcal{X})$. 
But $\varphi(A) \varphi\left(x_{2} \otimes f_{2}\right)+\varphi\left(x_{2} \otimes f_{2}\right) \varphi(A)=0$ and we get a contradiction. When $\mu-\lambda^{2}=0$ and $\lambda=0$, we know that $A^{2}=0$ and there exists a non-zero vector $x_{3} \in \mathcal{X}$ such that $x_{3}$ and $A x_{3}$ are linearly independent. Then there is an $f_{3} \in \mathcal{X}^{\prime}$ with $f_{3}\left(x_{3}\right)=0$ and $f_{3}\left(A x_{3}\right)=1$. Hence, $A x_{3} \otimes f_{3}+x_{3} \otimes f_{3} A \in \mathcal{I}^{*}(\mathcal{X})$ and so $\varphi(A) \varphi\left(x_{3} \otimes f_{3}\right)+\varphi\left(x_{3} \otimes\right.$ $\left.f_{3}\right) \varphi(A) \in \mathcal{I}^{*}(\mathcal{X})$. However, this contradicts $\varphi(A) \varphi\left(x_{3} \otimes f_{3}\right)+\varphi\left(x_{3} \otimes f_{3}\right) \varphi(A)=0$.

If $(A-\lambda)\left(A^{2}-\mu\right)=0$ and $\left(A^{2}-\mu\right)^{2}=0$, then $\left(\mu-\lambda^{2}\right) A^{2}=\lambda\left(\mu-\lambda^{2}\right)$. When $\mu-\lambda^{2} \neq 0$, we know that $A^{2}=\mu I$. Suppose $\mu \neq 0$. First, we assume that $A$ is a non-scalar operator. Then there is an $x_{4} \in \mathcal{X}$ such that $x_{4}$ and $A x_{4}$ are linearly independent. We can find $f_{4} \in \mathcal{X}^{\prime}$ with $f_{4}\left(x_{4}\right)=\frac{1}{2 \mu}$ and $f_{4}\left(A x_{4}\right)=0$. It follows $A\left(A x_{4}\right) \otimes f_{4}+\left(A x_{4}\right) \otimes f_{4} A \in \mathcal{I}^{*}(\mathcal{X})$, which implies $\varphi(A) \varphi\left(A x_{4} \otimes f_{4}\right)+\varphi\left(A x_{4} \otimes\right.$ $\left.f_{4}\right) \varphi(A)=0 \in \mathcal{I}^{*}(\mathcal{X})$. This gives a contradiction. Second, we assume $A=\beta I$ for some non-zero number $\beta$. For any pair $x_{5} \in \mathcal{X}$ and $f_{5} \in \mathcal{X}^{\prime}$ with $f_{5}\left(x_{5}\right)=\frac{1}{2 \beta}$, we have $A x_{5} \otimes f_{5}+x_{5} \otimes f_{5} A \in \mathcal{I}^{*}(\mathcal{X})$ and so $\varphi(A) \varphi\left(x_{5} \otimes f_{5}\right)+\varphi\left(x_{5} \otimes f_{5}\right) \varphi(A)=0 \in \mathcal{I}^{*}(\mathcal{X})$. This also gives a contradiction. Hence, $\mu=0$ and then $A^{2}=0$. Thus, there exists a nonzero vector $x_{6} \in \mathcal{X}$ such that $x_{6}$ and $A x_{6}$ are linearly independent. So, there is $f_{6} \in \mathcal{X}^{\prime}$ with $f_{6}\left(x_{6}\right)=0$ and $f_{6}\left(A x_{6}\right)=0$. Obviously, $A x_{6} \otimes f_{6}+x_{6} \otimes f_{6} A \in \mathcal{I}^{*}(\mathcal{X})$ and then $\varphi(A) \varphi\left(x_{6} \otimes f_{6}\right)+\varphi\left(x_{6} \otimes f_{6}\right) \varphi(A) \in \mathcal{I}^{*}(\mathcal{X})$. However, $\varphi(A) \varphi\left(x_{6} \otimes f_{6}\right)+\varphi\left(x_{6} \otimes f_{6}\right) \varphi(A)=$ 0 and this is also a contradiction. When $\mu-\lambda^{2}=0$, we know that $A^{3}-\lambda A^{2}-\lambda^{2} A+$ $\lambda^{3}=0$. Suppose $\lambda \neq 0$, we get $\frac{-1}{2 \lambda^{3}}\left(\left(A^{2}-\lambda A-\lambda^{2} I\right) A+A\left(A^{2}-\lambda A-\lambda^{2} I\right)\right)=I \in \mathcal{I}^{*}(\mathcal{X})$ implies $\frac{-1}{2 \lambda^{3}}\left(\varphi\left(A^{2}-\lambda A-\lambda^{2} I\right) \varphi(A)+\varphi(A) \varphi\left(A^{2}-\lambda A-\lambda^{2} I\right)\right)=0 \in \mathcal{I}^{*}(\mathcal{X})$. This is a contradiction. Suppose $\lambda=0$, we know $A^{3}=0$. If $A^{2} \neq 0$, then there is an $x_{7} \in \mathcal{X}$ such that $A x_{7}$ and $A^{2} x_{7}$ are linearly independent. So, there exists an $f_{7} \in \mathcal{X}^{\prime}$ such that $f_{7}\left(A x_{7}\right)=0$ and $f_{7}\left(A^{2} x_{7}\right)=1$. Hence, $A A x_{7} \otimes f_{7}+A x_{7} \otimes f_{7} A \in \mathcal{I}^{*}(\mathcal{X})$ and then $\varphi(A) \varphi\left(A x_{7} \otimes f_{7}\right)+\varphi\left(x_{7} \otimes f_{7}\right) \varphi(A)=0 \in \mathcal{I}^{*}(\mathcal{X})$. This is also a contradiction. If $A^{2}=0$, then we can derive another contradiction by using a routine argument demonstrated above.

Thus, $A=0$. Therefore, $\varphi$ is injective.

Lemma 3.2. Let $N \in \mathcal{B}(\mathcal{X})$ be of finite rank and $N^{2}=0$. Then $\varphi(N)$ is a nilpotent operator.

Proof. Note that $R(N)$ is finite dimensional. It is known that finite dimensional subspaces of a Banach space are complemented, and so $\mathcal{X}=R(N)+\mathcal{M}$ for some closed subspace $\mathcal{M}$ of $\mathcal{X}$ where $\mathcal{M}$ is a complementary subspace of $R(N)$. Then $N$ has the following operator matrix

$$
N=\left(\begin{array}{cc}
0 & N_{1} \\
0 & 0
\end{array}\right)
$$

Putting $P=\left(\begin{array}{ll}I & 0 \\ 0 & 0\end{array}\right)$, we have that $\left(\frac{1}{2}(P+z N)\right)(P+z N)+(P+z N)\left(\frac{1}{2}(P+z N)\right)=$ $P+z N \in \mathcal{I}^{*}(\mathcal{X})$ for all $z \in \mathbb{C}$. Thus, $\left(\frac{1}{2} \varphi(P+z N)\right) \varphi(P+z N)+\varphi(P+z N)\left(\frac{1}{2} \varphi(P+\right.$ 
$z N))=(\varphi(P)+z \varphi(N))^{2} \in \mathcal{I}^{*}(\mathcal{X})$ for all $z \in \mathbb{C}$. Hence, $(\varphi(P)+z \varphi(N))^{2}=(\varphi(P)+$ $z \varphi(N))^{4}$ for all $z \in \mathbb{C}$. That is,

$$
(\varphi(P))^{2}+z(\varphi(P) \varphi(N)+\varphi(N) \varphi(P))+z^{2}(\varphi(N))^{2}=(\varphi(P))^{4}+\cdots+z^{4}(\varphi(N))^{4}
$$

for all $z \in \mathbb{C}$. So, $(\varphi(N))^{4}=0$. $\square$

LEMma 3.3. If $\varphi$ is surjective, then $\varphi(I)=\lambda I$ for some constant $\lambda \in\{1,-1\}$.

Proof. Since $\varphi$ is a surjective map, there exists a non-zero operator $A \in \mathcal{B}(\mathcal{X})$ such that $\varphi(A)=I$. Assume that $A$ is a non-scalar operator. If there exists an $x \in \mathcal{X}$ such that $x, A x$ and $A^{2} x$ are linearly independent, then there is an $f \in \mathcal{X}^{\prime}$ such that $f(x)=f\left(A^{2} x\right)=0$ and $f(A x)=1$. So, $A x \otimes f+x \otimes f A \in \mathcal{I}^{*}(\mathcal{X})$, which implies that $\varphi(A) \varphi(x \otimes f)+\varphi(x \otimes f) \varphi(A)=2 \varphi(x \otimes f) \in \mathcal{I}^{*}(\mathcal{X})$. But $2 \varphi(x \otimes f)$ is a nilpotent operator by Lemma 3.2 and this contradiction implies that $x, A x$ and $A^{2} x$ are linearly dependent for every $x \in \mathcal{X}$. Then there exist scalars $\lambda$ and $\mu$ such that $(A-\lambda)\left(A^{2}-\mu\right)=0$ and either $(A-\lambda)^{2}=0$ or $\left(A^{2}-\mu\right)^{2}=0$ by Lemma 2.2.

If $(A-\lambda)\left(A^{2}-\mu\right)=0$ and $(A-\lambda)^{2}=0$, then $\left(\mu-\lambda^{2}\right) A=\lambda\left(\mu-\lambda^{2}\right)$. Since $A$ is a non-scalar operator, we know that $\mu=\lambda^{2}$. When $\lambda=0$, we get that $A^{2}=$ 0 and there exist $x_{1} \in \mathcal{X}$ and $f_{1} \in \mathcal{X}^{\prime}$ such that $f_{1}\left(x_{1}\right)=0$ and $f_{1}\left(A x_{1}\right)=1$. So, $A\left(x_{1} \otimes f_{1}\right)+\left(x_{1} \otimes f_{1}\right) A \in \mathcal{I}^{*}(\mathcal{X})$ implies $\varphi(A) \varphi\left(x_{1} \otimes f_{1}\right)+\varphi\left(x_{1} \otimes f_{1}\right) \varphi(A)=$ $2 \varphi\left(x_{1} \otimes f_{1}\right) \in \mathcal{I}^{*}(\mathcal{X})$. However, $2 \varphi\left(x_{1} \otimes f_{1}\right)$ is a nilpotent operator by Lemma 3.2 . This contradiction implies that $\lambda \neq 0$ and $A-\lambda$ is a nilpotent operator. Then there is a non-zero vector $x_{2} \in \mathcal{X}$ such that $(A-\lambda) x_{2}=0$. For every $f_{2} \in \mathcal{X}^{\prime}$ with $f_{2}\left(x_{2}\right)=\frac{1}{2 \lambda}$, we know that $A x_{2} \otimes f_{2}+x_{2} \otimes f_{2} A \in \mathcal{I}^{*}(\mathcal{X})$, which implies that $\varphi(A) \varphi\left(x_{2} \otimes f_{2}\right)+\varphi\left(x_{2} \otimes f_{2}\right) \varphi(A)=2 \varphi\left(x_{2} \otimes f_{2}\right) \in \mathcal{I}^{*}(\mathcal{X})$. Moreover, $2 \lambda \varphi\left(x_{2} \otimes\right.$ $\left.f_{2}\right) \in \mathcal{I}^{*}(\mathcal{X})$ follows from $2 \lambda x_{2} \otimes f_{2} \in \mathcal{I}^{*}(\mathcal{X})$. Hence, $\lambda^{2}=1$. When $\lambda=1$, we know that $A^{2}-2 A+I=0$ and so $(2 I-A) \frac{A}{2}+\frac{A}{2}(2 I-A)=I \in \mathcal{I}^{*}(\mathcal{X})$. Then, $\varphi(2 I-A) \varphi\left(\frac{A}{2}\right)+\varphi\left(\frac{A}{2}\right) \varphi(2 I-A)=2 \varphi(I)-I \in \mathcal{I}^{*}(\mathcal{X})$. Setting $2 \varphi(I)-I=R \in \mathcal{I}^{*}(\mathcal{X})$, we get that $\varphi(I)=\frac{I+R}{2}$. However, $I \frac{I}{2}+\frac{I}{2} I=I \in \mathcal{I}^{*}(\mathcal{X})$ implies $\varphi(I)^{2} \in \mathcal{I}^{*}(\mathcal{X})$. Thus, $\varphi(I)^{2}=\frac{I+3 R}{4}$ and so $R=I$. It follows that $\varphi(I)=I=\varphi(A)$ and then $A=I$ by the injection of $\varphi$. This is a contradiction. Similarly we can also obtain a contradiction when $\lambda=-1$.

If $(A-\lambda)\left(A^{2}-\mu\right)=0$ and $\left(A^{2}-\mu\right)^{2}=0$, then $\left(\mu-\lambda^{2}\right) A^{2}=\lambda\left(\mu-\lambda^{2}\right)$. When $\mu-\lambda^{2} \neq 0$, we get $A^{2}=\lambda I$. When $\lambda \neq 0$, there is a vector $x_{4} \in \mathcal{X}$ such that $x_{4}$ and $A x_{4}$ are linearly independent. Selecting $f_{4} \in \mathcal{X}^{\prime}$ with $f_{4}\left(x_{4}\right)=\frac{1}{\mu}$ and $f_{4}\left(A x_{4}\right)=0$, we know that $A A x_{4} \otimes f_{4}+A x_{4} \otimes f_{4} A \in \mathcal{I}^{*}(\mathcal{X})$ and so $2 \varphi\left(A x_{4} \otimes f_{4}\right) \in \mathcal{I}^{*}(\mathcal{X})$. However, $\varphi\left(A x_{4} \otimes f_{4}\right)$ is a nilpotent operator. This contradiction shows that $\mu=0$ and then $A^{2}=0$. Since $x_{4}$ and $A x_{4}$ are linearly independent, there is an $f_{5} \in \mathcal{X}^{\prime}$ with $f_{5}\left(x_{4}\right)=0$ and $f_{5}\left(A x_{4}\right)=1$. So, $A x_{4} \otimes f_{5}+x_{4} \otimes f_{5} A \in \mathcal{I}^{*}(\mathcal{X})$ and then $2 \varphi\left(x_{4} \otimes f_{5}\right) \in \mathcal{I}^{*}(\mathcal{X})$. But this contradicts with that $\varphi\left(x_{4} \otimes f_{5}\right)$ is a nilpotent operator. When $\mu-\lambda^{2}=0$ and $\lambda=0$, we know $A^{3}=0$. For the case that $A^{2}=0$, we also get 
a contradiction using a similar argument above. For the case that $A^{2} \neq 0$, there is an $x_{6} \in \mathcal{X}$ such that $A x_{6}$ and $A^{2} x_{6}$ are linearly independent. Selecting $f_{6} \in \mathcal{X}^{\prime}$ such that $f_{6}\left(A x_{6}\right)=0$ and $f_{6}\left(A^{2} x_{6}\right)=1$, we know $A A x_{6} \otimes f_{6}+A x_{6} \otimes f_{6} A \in \mathcal{I}^{*}(\mathcal{X})$ and then $2 \varphi\left(A x_{6} \otimes f_{6}\right) \in \mathcal{I}^{*}(\mathcal{X})$. This contradicts the fact that $\varphi\left(A x_{6} \otimes f_{6}\right)$ is a nilpotent operator. So, $\lambda \neq 0$ and $A^{2}-\lambda^{2}$ is a nilpotent operator. Hence, there is an $x_{7} \in \mathcal{X}$ such that $A^{2} x_{7}=\lambda^{2} x_{7}$. If $x_{7}$ and $A x_{7}$ are linearly independent, then there is an $f_{7} \in \mathcal{X}^{\prime}$ such that $f_{7}\left(x_{7}\right)=\frac{1}{\lambda^{2}}$ and $f_{7}\left(A x_{7}\right)=0$. It follows that $A A x_{7} \otimes f_{7}+A x_{7} \otimes f_{7} A \in \mathcal{I}^{*}(\mathcal{X})$ which gives that $2 \varphi\left(A x_{7} \otimes f_{7}\right) \in \mathcal{I}^{*}(\mathcal{X})$. While $\varphi\left(A x_{7} \otimes f_{7}\right)$ is a nilpotent operator, we get a contradiction. If $A x_{7}=\alpha x_{7}$ for some $\alpha \in \mathbb{C}$, then $\alpha^{2}=\lambda^{2}$. Selecting $f_{8} \in \mathcal{X}^{\prime}$ such that $f_{8}\left(x_{7}\right)=\frac{1}{2 \lambda^{2}}$, we get that $f_{8}\left(A x_{7}\right)=\frac{\alpha}{2 \lambda^{2}}$. By direct calculation, we know that $\left(2 \lambda^{2}\left(A x_{7} \otimes f_{8}\right)\right)\left(A x_{7} \otimes f_{8}\right)+\left(A x_{7} \otimes f_{8}\right)\left(2 \lambda^{2}\left(A x_{7} \otimes f_{8}\right)\right) \in \mathcal{I}^{*}(\mathcal{X})$ and so $4 \lambda^{2} \varphi\left(A x_{7} \otimes f_{8}\right)^{2} \in \mathcal{I}^{*}(\mathcal{X})$. Moreover, $A A x_{7} \otimes f_{8}+A x_{7} \otimes f_{8} A \in \mathcal{I}^{*}(\mathcal{X})$ implies that $2 \varphi\left(A x_{7} \otimes f_{8}\right) \in \mathcal{I}^{*}(\mathcal{X})$. It follows that $\lambda^{2}=1$. Since $I=2 A^{2}-A^{4}=$ $\left(2 I-A^{2}\right)\left(\frac{A^{2}}{2}\right)+\left(\frac{A^{2}}{2}\right)\left(2 I-A^{2}\right)=\left(2 A-A^{3}\right)\left(\frac{A}{2}\right)+\left(\frac{A}{2}\right)\left(2 A-A^{3}\right)$, we have that $\varphi\left(2 I-A^{2}\right) \varphi\left(\frac{A^{2}}{2}\right)+\varphi\left(\frac{A^{2}}{2}\right) \varphi\left(2 I-A^{2}\right) \in \mathcal{I}^{*}(\mathcal{X})$ and $2 I-\varphi\left(A^{3}\right) \in \mathcal{I}^{*}(\mathcal{X})$. On the one hand, $\varphi\left(2 I-A^{2}\right) \varphi\left(\frac{A^{2}}{2}\right)+\varphi\left(\frac{A^{2}}{2}\right) \varphi\left(2 I-A^{2}\right)=\varphi(I) \varphi\left(A^{2}\right)+\varphi\left(A^{2}\right) \varphi(I)-\varphi\left(A^{2}\right)^{2} \in$ $\mathcal{I}^{*}(\mathcal{X})$. On the other hand, $A^{3}-A-\lambda A^{2}+\lambda=0$ implies $I=\frac{1}{\lambda} A+A^{2}-\frac{1}{\lambda} A^{3}=$ $\left(\frac{1}{\lambda} I+A-\frac{1}{\lambda} A^{2}\right)\left(\frac{A}{2}\right)+\left(\frac{A}{2}\right)\left(\frac{1}{\lambda} I+A-\frac{1}{\lambda} A^{2}\right)$. Hence, $\frac{1}{\lambda}\left[\varphi\left(A^{2}\right)-\varphi(I)\right]=I-E$ for some $E \in \mathcal{I}^{*}(\mathcal{X})$. It gives $\varphi\left(A^{2}\right)^{2}-\left[\varphi(I) \varphi\left(A^{2}\right)+\varphi\left(A^{2}\right) \varphi(I)\right]+\varphi(I)^{2}=I-E$. Furthermore, $\varphi\left(A^{3}\right)-I=\lambda\left[\varphi(I)-\varphi\left(A^{2}\right)\right]$ implies $\left(\varphi\left(A^{3}\right)-I\right)^{2}=\varphi(I)^{2}-\left[\varphi(I) \varphi\left(A^{2}\right)+\varphi\left(A^{2}\right) \varphi(I)\right]+$ $\varphi\left(A^{2}\right)^{2}$. It follows $\varphi\left(A^{3}\right)^{2}-2 \varphi\left(A^{3}\right)+I=I-E$ and $\varphi\left(A^{3}\right)^{2}-2 \varphi\left(A^{3}\right)=-E$. However, $\left(2 I-\varphi\left(A^{3}\right)\right)^{2}=4 I-4 \varphi\left(A^{3}\right)+\varphi\left(A^{3}\right)^{2}=2 I-\varphi\left(A^{3}\right)$ implies $\varphi\left(A^{3}\right)=2 I-E$. So, $\frac{1}{\lambda}\left[\varphi\left(A^{2}\right)-\varphi(I)\right]=I-E=I+\varphi\left(A^{3}\right)$ and then $\varphi\left(\lambda A^{3}+\lambda A-A^{2}+I\right)=0$. Using the fact that $\varphi$ is a bijection, we get $\lambda A^{3}+\lambda A-A^{2}+I=0$. Noting that $A^{3}-A-\lambda A^{2}+\lambda=0$, we know $A=0$ and this is a contradiction.

Therefore, $A=\mu I$ for some non-zero complex number $\mu$. We thus have that $\varphi(I)=\lambda I$ for some constant $\lambda \in \mathbb{C}$ and $\lambda^{2}=1$.

Next we assume that $\varphi$ is surjective and $\varphi(I)=I$. We may replace $\varphi$ by $-\varphi$ if $\varphi(I)=-I$.

LEMMA 3.4. $\varphi$ has the following properties:

(1) $\varphi(\mathcal{I}(\mathcal{X})) \subseteq \mathcal{I}(\mathcal{X})$;

(2) $\varphi$ preserves the orthogonality of idempotents;

(3) $\varphi$ preserves the order of idempotents.

Proof. (1) It follows directly from $\varphi(I)=I, \varphi(0)=0$ and the fact that $\left(\frac{1}{2} P\right) I+$ $I\left(\frac{1}{2} P\right)=P$ for any $P \in \mathcal{I}^{*}(\mathcal{X})$.

(2) If $P, Q \in \mathcal{I}^{*}(\mathcal{X})$ and $P \perp Q$, then $P+Q \in \mathcal{I}^{*}(\mathcal{X})$. So, $\varphi(P+Q)=\varphi(P)+$ $\varphi(Q) \in \mathcal{I}^{*}(\mathcal{X})$ by $(1)$. Since $\varphi(P), \varphi(Q) \in \mathcal{I}^{*}(\mathcal{X})$, we know that $\varphi(P) \perp \varphi(Q)$. 
(3) Let $P, Q \in \mathcal{I}^{*}(\mathcal{X})$ and $P<Q$. Then $P Q=Q P=P$ and $Q=P+(Q-P)$. Clearly, $P \in \mathcal{I}^{*}(\mathcal{X})$ and $Q-P \in \mathcal{I}^{*}(\mathcal{X})$. Thus, $\varphi(Q)-\varphi(P) \in \mathcal{I}^{*}(\mathcal{X})$, and we get $2 \varphi(P)=\varphi(Q) \varphi(P)+\varphi(P) \varphi(Q)$. This implies that $\varphi(Q) \varphi(P)=\varphi(P) \varphi(Q)$ $=\varphi(P)$.

Lemma 3.5. $\varphi\left(\mathcal{I}_{1}(\mathcal{X})\right) \subseteq \mathcal{I}_{1}(\mathcal{X})$.

Proof. Let $P=x \otimes f \in \mathcal{I}_{1}(\mathcal{X})$ for some $x \in \mathcal{X}$ and $f \in \mathcal{X}^{\prime}$ with $f(x)=1$. Then $\varphi(P) \in \mathcal{I}^{*}(\mathcal{X})$ by Lemma 3.4. Assume that $\operatorname{rank} \varphi(P) \geq 2$. Then there exists a $R \in \mathcal{I}_{1}(\mathcal{X})$ such that $R<\varphi(P)$ and so $\varphi(P)-R \in \mathcal{I}^{*}(\mathcal{X})$. Since $\varphi$ is bijective, there is a non-zero operator $B \in \mathcal{B}(\mathcal{X})$ such that $R=\varphi(B)$.

If $x, B x$ and $B^{2} x$ are linearly independent, there is a $g \in \mathcal{X}^{\prime}$ such that $g(B x)=1-$ $f(B x), g\left(B^{2} x\right)=1-f\left(B^{2} x\right)$ and $g(x)=-1$. Then $B x \otimes(f+g)+x \otimes(f+g) B \in \mathcal{I}^{*}(\mathcal{X})$ implies $\varphi(B) \varphi(x \otimes(f+g))+\varphi(x \otimes(f+g)) \varphi(B)=2 R+R \varphi(x \otimes g)+\varphi(x \otimes g) R \in \mathcal{I}^{*}(\mathcal{X})$. On the one hand, $-x \otimes g \in \mathcal{I}^{*}(\mathcal{X})$ implies $-\varphi(x \otimes g) \in \mathcal{I}^{*}(\mathcal{X})$, and $z x \otimes f \cdot(-x) \otimes g+(1-$ $z)(-x) \otimes g \cdot x \otimes f \in \mathcal{I}^{*}(\mathcal{X})$ implies $z \varphi(x \otimes f) \cdot \varphi((-x) \otimes g)+(1-z) \varphi((-x) \otimes g) \varphi(x \otimes f) \in$ $\mathcal{I}^{*}(\mathcal{X})$ for all $z \in \mathbb{C}$. On the other hand, $z x \otimes f \cdot(-x) \otimes g+(1-z)(-x) \otimes g \cdot x \otimes f=$ $z(-x) \otimes g+(1-z) x \otimes f \in \mathcal{I}^{*}(\mathcal{X})$ implies $z \varphi((-x) \otimes g)+(1-z) \varphi(x \otimes f) \in \mathcal{I}^{*}(\mathcal{X})$ for all $z \in \mathbb{C}$. Hence, $\varphi((-x) \otimes g) \varphi(x \otimes f), \varphi(x \otimes f) \varphi((-x) \otimes g) \in \mathcal{I}^{*}(\mathcal{X})$ and

$$
\begin{aligned}
& \varphi(x \otimes f) \varphi((-x) \otimes g) \varphi(x \otimes f)+\varphi((-x) \otimes g) \varphi(x \otimes f) \varphi((-x) \otimes g) \\
& =\varphi(x \otimes f) \varphi((-x) \otimes g)+\varphi((-x) \otimes g) \varphi(x \otimes f) \\
& =\varphi(x \otimes f)+\varphi((-x) \otimes g) .
\end{aligned}
$$

Under the decomposition $\mathcal{X}=R(R)+(R(P) \ominus N(R))+N(P), R, P$ and $-\varphi(x \otimes g)$ have the following operator matrices

$$
R=\left(\begin{array}{lll}
1 & 0 & 0 \\
0 & 0 & 0 \\
0 & 0 & 0
\end{array}\right), P=\left(\begin{array}{ccc}
1 & 0 & 0 \\
0 & 1 & 0 \\
0 & 0 & 0
\end{array}\right) \text { and } \varphi(-x \otimes g)=\left(\begin{array}{lll}
Q_{11} & Q_{12} & Q_{13} \\
Q_{21} & Q_{22} & Q_{23} \\
Q_{31} & Q_{32} & Q_{33}
\end{array}\right)
$$

respectively. By direct calculation, we know

$$
\left(\begin{array}{ccc}
Q_{11} & Q_{12} & Q_{13} \\
Q_{21} & Q_{22} & Q_{23} \\
0 & 0 & 0
\end{array}\right)+\left(\begin{array}{lll}
Q_{11} & Q_{12} & 0 \\
Q_{21} & Q_{22} & 0 \\
Q_{31} & Q_{32} & 0
\end{array}\right)=\left(\begin{array}{ccc}
1+Q_{11} & Q_{12} & Q_{13} \\
Q_{21} & 1+Q_{22} & Q_{23} \\
Q_{31} & Q_{32} & Q_{33}
\end{array}\right) .
$$

So, $Q_{11}=1, Q_{12}=0, Q_{21}=0, Q_{33}=0$ and $Q_{22}=1$. It follows that

$$
2 R+R \varphi(x \otimes g)+\varphi(x \otimes g) R=\left(\begin{array}{ccc}
0 & 0 & -Q_{13} \\
0 & 0 & 0 \\
-Q_{31} & 0 & 0
\end{array}\right) .
$$

Hence, we get a contradiction since $2 R+R \varphi(x \otimes g)+\varphi(x \otimes g) R \in \mathcal{I}^{*}(\mathcal{X})$. 
If $x, B x$ and $B^{2} x$ are linearly dependent and $x$ and $B x$ are linearly independent, then $B^{2} x=\lambda x+\mu B x$ for some $\lambda, \mu \in \mathbb{C}$. When $\mu=0$, there is $h \in \mathcal{X}^{\prime}$ such that $h(B x)=1-f(B x)$ and $h(x)=-1$. Then we know that $B x \otimes(f+h)+x \otimes(f+h) B \in$ $\mathcal{I}^{*}(\mathcal{X})$ implies $\varphi(B) \varphi(x \otimes(f+h))+\varphi(x \otimes(f+h)) \varphi(B)=2 R+R \varphi(x \otimes h)+\varphi(x \otimes h) R \in$ $\mathcal{I}^{*}(\mathcal{X})$. Note that $-x \otimes h \in \mathcal{I}^{*}(\mathcal{X})$ and $z x \otimes f \cdot(-x) \otimes h+(1-z)(-x) \otimes h \cdot x \otimes f=$ $z(-x) \otimes h+(1-z) x \otimes f \in \mathcal{I}^{*}(\mathcal{X})$. So, we can get another contradiction using a similar argument demonstrated above. Now, we assume that $\mu \neq 0$. If $\lambda=0$, then $B^{2} x=\mu B x$. Thus, there is $h_{1} \in \mathcal{X}^{\prime}$ such that $h_{1}(B x)=\frac{\mu}{2}-f(B x)$ and $h_{1}(x)=-1$. It follows that $\left(I-\frac{2}{\mu} B\right) x \otimes\left(f+h_{1}\right)+x \otimes\left(f+h_{1}\right)\left(I-\frac{2}{\mu} B\right) \in \mathcal{I}^{*}(\mathcal{X})$ and then

$$
\begin{aligned}
& \varphi\left(I-\frac{2}{\mu} B\right) \varphi\left(x \otimes\left(f+h_{1}\right)\right)+\varphi\left(x \otimes\left(f+h_{1}\right)\right) \varphi\left(I-\frac{2}{\mu} B\right) \\
& =2 P-\frac{4}{\mu} R-2 \varphi\left(-x \otimes h_{1}\right)+\frac{2}{\mu}\left[\varphi\left(-x \otimes h_{1}\right) R+R \varphi\left(-x \otimes h_{1}\right)\right] \in \mathcal{I}^{*}(\mathcal{X}) .
\end{aligned}
$$

Note that $-x \otimes h_{1} \in \mathcal{I}^{*}(\mathcal{X})$ and $z x \otimes f \cdot(-x) \otimes h_{1}+(1-z)(-x) \otimes h_{1} \cdot x \otimes f=z(-x) \otimes$ $h_{1}+(1-z) x \otimes f \in \mathcal{I}^{*}(\mathcal{X})$. We get that $\left.-\varphi\left(x \otimes h_{1}\right) \in \mathcal{I}^{*}(\mathcal{X}), z \varphi(x \otimes f) \cdot \varphi(-x) \otimes h_{1}\right)+$ $(1-z) \varphi\left((-x) \otimes h_{1}\right) \varphi(x \otimes f) \in \mathcal{I}^{*}(\mathcal{X})$ for all $z \in \mathbb{C}$ and $\left.z \varphi(-x) \otimes h_{1}\right)+(1-z) \varphi(x \otimes f) \in$ $\mathcal{I}^{*}(\mathcal{X})$ for all $z \in \mathbb{C}$. Under the decomposition $\mathcal{X}=R(R)+(R(P) \ominus N(R))+N(P)$, $R, P$ and $-\varphi\left(x \otimes h_{1}\right)$ have the following operator matrices

$R=\left(\begin{array}{lll}1 & 0 & 0 \\ 0 & 0 & 0 \\ 0 & 0 & 0\end{array}\right), P=\left(\begin{array}{lll}1 & 0 & 0 \\ 0 & 1 & 0 \\ 0 & 0 & 0\end{array}\right)$ and $\varphi\left(-x \otimes h_{1}\right)=\left(\begin{array}{lll}Q_{11} & Q_{12} & Q_{13} \\ Q_{21} & Q_{22} & Q_{23} \\ Q_{31} & Q_{32} & Q_{33}\end{array}\right)$

respectively. By direct calculation, we know that

$$
\left(\begin{array}{ccc}
Q_{11} & Q_{12} & Q_{13} \\
Q_{21} & Q_{22} & Q_{23} \\
0 & 0 & 0
\end{array}\right)+\left(\begin{array}{ccc}
Q_{11} & Q_{12} & 0 \\
Q_{21} & Q_{22} & 0 \\
Q_{31} & Q_{32} & 0
\end{array}\right)=\left(\begin{array}{ccc}
1+Q_{11} & Q_{12} & Q_{13} \\
Q_{21} & 1+Q_{22} & Q_{23} \\
Q_{31} & Q_{32} & Q_{33}
\end{array}\right) .
$$

So, $Q_{11}=1, Q_{12}=0, Q_{21}=0, Q_{33}=0$ and $Q_{22}=1$. It follows that

$$
\begin{aligned}
& \varphi\left(I-\frac{2}{\mu} B\right) \varphi\left(x \otimes\left(f+h_{1}\right)\right)+\varphi\left(x \otimes\left(f+h_{1}\right)\right) \varphi\left(I-\frac{2}{\mu} B\right) \\
& =2 P-\frac{4}{\mu} R-2 \varphi\left(-x \otimes h_{1}\right)+\frac{2}{\mu}\left[\varphi\left(-x \otimes h_{1}\right) R+R \varphi\left(-x \otimes h_{1}\right)\right] \in \mathcal{I}^{*}(\mathcal{X}) \\
& =\left(\begin{array}{ccc}
0 & 0 & \left(-2+\frac{2}{\mu}\right) Q_{13} \\
0 & 0 & -2 Q_{23} \\
\left(-2+\frac{2}{\mu}\right) Q_{31} & -2 Q_{32} & 0
\end{array}\right) \in \mathcal{I}^{*}(\mathcal{X}) .
\end{aligned}
$$

Clearly, this is a contradiction. If $\lambda \neq 0$, then there is $h_{2} \in \mathcal{X}^{\prime}$ such that $h_{2}(B x)=$ $\frac{-\mu}{2}-f(B x)$ and $h_{2}(x)=-f(x)$. Hence, $\left(I-\frac{2}{\mu} B\right) x \otimes\left(f+h_{2}\right)+x \otimes\left(f+h_{2}\right)\left(I-\frac{2}{\mu} B\right) \in$ $\mathcal{I}^{*}(\mathcal{X})$, and thus,

$$
\begin{aligned}
& \varphi\left(I-\frac{2}{\mu} B\right) \varphi\left(x \otimes\left(f+h_{2}\right)\right)+\varphi\left(x \otimes\left(f+h_{2}\right)\right) \varphi\left(I-\frac{2}{\mu} B\right) \\
& =2 P-\frac{4}{\mu} R-2 \varphi\left(-x \otimes h_{2}\right)+\frac{2}{\mu}\left[\varphi\left(-x \otimes h_{2}\right) R+R \varphi\left(-x \otimes h_{2}\right)\right] \in \mathcal{I}^{*}(\mathcal{X}) .
\end{aligned}
$$


By an argument similar to that above, we also get a contradiction.

If $x, B x$ and $B^{2} x$ are linearly dependent and $x$ and $B x$ are linearly dependent, then $B x=\beta x$ for some $\beta \in \mathbb{C}$. When $\beta=0$, we know that $\frac{1}{2}((I+z B) x \otimes f+x \otimes$ $f(I+z B)) \in \mathcal{I}^{*}(\mathcal{X})$ for all $z \in \mathbb{C} \backslash\{0\}$ implies that $\frac{1}{2}((I+z R) P+P(I+z R))=$ $P+z R \in \mathcal{I}^{*}(\mathcal{X})$ for all $z \in \mathbb{C} \backslash\{0\}$. Obviously, this is a contradiction. When $\beta \neq 0$, we know that $\frac{1}{2(1+\beta z)}((I+z B) x \otimes f+x \otimes f(I+z B)) \in \mathcal{I}^{*}(\mathcal{X})$ for all $z \in \mathbb{C} \backslash\{0\}$ with $1+\beta z \neq 0$ implies that $\frac{1}{2(1+\beta z)}((I+z R) P+P(I+z R))=\frac{1}{(1+\beta z)}(P+z R) \in \mathcal{I}^{*}(\mathcal{X})$ for all $z \in \mathbb{C} \backslash\{0\}$ with $1+\beta z \neq 0$. This is also a contradiction.

Therefore, $\varphi(P) \in \mathcal{I}_{1}(\mathcal{X})$.

The proof of the following lemma is similar to that of [13, Lemma 2.7 ].

Lemma 3.6. $\varphi\left(\mathcal{N}_{1}(\mathcal{X})\right) \subseteq \mathcal{N}_{1}(\mathcal{X})$.

Proof. Let $N=x \otimes f \in \mathcal{N}_{1}(\mathcal{X})$ for some non-zero $x \in \mathcal{X}$ and non-zero $f \in \mathcal{X}^{\prime}$ such that $f(x)=0$. Then $\varphi(N) \in \mathcal{N}(\mathcal{X})$ by Lemma 3.2. Taking an $f_{1} \in \mathcal{X}^{\prime}$ such that $f_{1}(x)=1$ and setting $Q=x \otimes f_{1}$, we know that both $Q$ and $Q+N$ are in $\mathcal{I}_{1}(\mathcal{X})$. So are both $\varphi(Q)$ and $\varphi(Q+N)$ by Lemma 3.5. Then there exist $y_{1}, y_{2} \in \mathcal{X}$ and $g_{1}, g_{2} \in \mathcal{X}^{\prime}$ such that $g_{1}\left(y_{1}\right)=g_{2}\left(y_{2}\right)=1, \varphi(Q)=y_{1} \otimes g_{1}$ and $\varphi(Q+N)=y_{2} \otimes g_{2}$. Putting $P=\frac{1}{2}((Q+N)+Q)=\frac{1}{2} N+Q$, we get that $P \in \mathcal{I}_{1}(\mathcal{X})$ and then $\varphi(P) \in \mathcal{I}_{1}(\mathcal{X})$. However, $\varphi(P)=\frac{1}{2}(\varphi(Q+N)+\varphi(Q))=\frac{1}{2}\left(y_{1} \otimes g_{1}+y_{2} \otimes g_{2}\right)$. It follows that either $y_{1}$ and $y_{2}$ or $g_{1}$ and $g_{2}$ are linearly dependent. If $y_{1}$ and $y_{2}$ are linearly dependent, then we may assume that $y_{1}=y_{2}$. Thus, $\varphi(P)=\frac{1}{2} y_{1} \otimes\left(g_{1}+g_{2}\right) \in \mathcal{I}_{1}(\mathcal{X})$ and then $g_{1}\left(y_{1}\right)+g_{2}\left(y_{1}\right)=2$. Since $g_{1}\left(y_{1}\right)=1$, we have $g_{2}\left(y_{1}\right)=1$. Thus, $\varphi(N)=$ $\varphi(N+Q)-\varphi(Q)=y_{1} \otimes\left(g_{1}-g_{2}\right)$ and $\left(g_{1}-g_{2}\right)\left(y_{1}\right)=0$. Hence, $\varphi(N) \in \mathcal{N}_{1}(\mathcal{X})$. We can get that $\varphi(N) \in \mathcal{N}_{1}(\mathcal{X})$ by similar discussion if $g_{1}$ and $g_{2}$ are linearly dependent.

Corollary 3.7. $\varphi\left(\mathcal{F}_{1}(\mathcal{X})\right) \subseteq \mathcal{F}_{1}(\mathcal{X})$ and $\varphi(\mathcal{F}(\mathcal{X})) \subseteq \mathcal{F}(\mathcal{X})$

Proof. Since every non-nilpotent rank-one operator is a non-zero scalar multiple of rank-one idempotent operator, we know that $\varphi\left(\mathcal{F}_{1}(\mathcal{X})\right) \subseteq \mathcal{F}_{1}(\mathcal{X})$ by Lemma 3.5 and Lemma 3.6 and the linearity of $\varphi$. Moreover, every finite-rank operator can be written as a linear combination of finitely many rank-one operators. It follows from the linearity of $\varphi$ that $\varphi(\mathcal{F}(\mathcal{X})) \subseteq \mathcal{F}(\mathcal{X})$.

Note that a linear map $\varphi$ on $\mathcal{F}(\mathcal{X})$ is rank non-increasing if $\operatorname{rank} \varphi(X) \leq \operatorname{rank} X$ for any $X \in \mathcal{F}(\mathcal{X})$. We start this section with [5, Corollary 2.1.5], which is restated in the following.

LEMMA 3.8. Let $\varphi$ be a linear map on $\mathcal{F}(\mathcal{X})$ which is rank non-increasing such that $\operatorname{rank} \varphi\left(T_{0}\right)>1$ for some $T_{0} \in \mathcal{F}(\mathcal{X})$. Then one of the following holds. 
(1) There exist linear injective maps $A: \mathcal{X} \rightarrow \mathcal{X}$ and $C: \mathcal{X}^{\prime} \rightarrow \mathcal{X}^{\prime}$ such that $\varphi(x \otimes f)=A x \otimes C f$ for all $x \in \mathcal{X}$ and $f \in \mathcal{X}^{\prime}$.

(2) There exist linear injective maps $A: \mathcal{X}^{\prime} \rightarrow \mathcal{X}$ and $C: \mathcal{X} \rightarrow \mathcal{X}^{\prime}$ such that $\varphi(x \otimes f)=A f \otimes C x$ for all $x \in \mathcal{X}$ and $f \in \mathcal{X}^{\prime}$.

Proposition 3.9. Let $\varphi$ be a surjective linear map on $\mathcal{B}(\mathcal{X})$ preserving the nonzero idempotency of Jordan products of two operators such that $\varphi(I)=I$. Then one of the statements in Lemma 3.8 holds.

Proof. From Corollary 3.7, we know that the restriction $\left.\varphi\right|_{\mathcal{F}(\mathcal{X})}$ of $\varphi$ on $\mathcal{F}(\mathcal{X})$ is a linear map preserving rank non-increasing. Let $Q$ be a rank-2 idempotent operator. Then there exists a $P \in \mathcal{I}_{1}(\mathcal{X})$ such that $P<Q$. By Lemma 3.4, we know that $\varphi(P)<\varphi(Q)$ and $\varphi(P) \in \mathcal{I}_{1}(\mathcal{X})$. So, $\operatorname{rank} \varphi(Q) \geq 2$. Then we get the desired results from Lemma 3.8.

Proof of Theorem 1.1. The sufficiency is clear. Let $\varphi$ be a linear map on $\mathcal{M}_{n}$ preserving the nonzero idempotency of Jordan product of two operators. Then $\varphi$ is injective by Lemma 3.1 and thus bijective. We now have $\varphi(I)=\lambda I$ for some constant $\lambda \in\{1,-1\}$. we may assume $\varphi(I)=I$. Then one of two statements in Proposition 3.1 holds.

If (1) holds, then we easily have that $\varphi(X)=A X B$ for all $X \in \mathcal{M}_{n}$. It is clear that $B=A^{-1}$. We have that $\varphi(X)=A X A^{-1}$ for all $X \in \mathcal{M}_{n}$.

If (2) holds, then we similarly have that $\varphi(X)=A X^{t} A^{-1}$ for all $X \in \mathcal{M}_{n}$.

We next consider the infinite dimensional case.

LEMma 3.10. Let $\varphi$ be a surjective linear map on $\mathcal{B}(\mathcal{X})$ preserving the nonzero idempotency of Jordan products of two operators such that $\varphi(I)=I$. Then $\varphi\left(\mathcal{F}_{1}(\mathcal{X})\right)$ $=\mathcal{F}_{1}(\mathcal{X})$.

Proof. By Corollary 3.7, it is sufficient to prove that $\mathcal{F}_{1}(\mathcal{X}) \subseteq \varphi\left(\mathcal{F}_{1}(\mathcal{X})\right)$. Let $T \in$ $\mathcal{B}(\mathcal{H})$ such that $\varphi(T)=z \otimes h$ is of rank-one for some $z \in \mathcal{X}$ and $h \in \mathcal{X}^{\prime}$. Clearly, $T$ is a non-scalar operator. So, there is $x \in \mathcal{X}$ such that $x$ and $T x$ are linearly independent. If $\operatorname{rank} T>1$, then there exists $y \in \mathcal{X}$ such that $T x$ and $T y$ are linearly independent. It follows that $x$ and $y$ are linearly independent. Applying [9, Lemma 2.1], we know that $x+\delta y$ and $T(x+\delta y)$ are linearly independent for all but finite number $\delta \in \mathbb{C}$. For any fixed $\alpha \in \mathbb{C}$, there is a rank-one idempotent $P(\delta, \alpha)$ such that $(T+P(\delta, \alpha))(x+\delta y)=$ $\alpha(x+\delta y)$ by Lemma 2.4. Finding $f_{(\delta, \alpha)} \in \mathcal{X}^{\prime}$ to satisfy $f_{(\delta, \alpha)}(x+\delta y)=\frac{1}{2 \alpha}$, we get that $\frac{1}{2 \alpha}\left((T+P(\delta, \alpha))(x+\delta y) \otimes f_{(\delta, \alpha)}+(x+\delta y) \otimes f_{(\delta, \alpha)}(T+P(\delta, \alpha))\right) \in \mathcal{I}^{*}(\mathcal{X})$, which implies that $\frac{1}{2 \alpha}\left(\varphi(T+P(\delta, \alpha)) \varphi\left((x+\delta y) \otimes f_{(\delta, \alpha)}\right)+\varphi\left((x+\delta y) \otimes f_{(\delta, \alpha)}\right) \varphi(T+\right.$ $P(\delta, \alpha))) \in \mathcal{I}^{*}(\mathcal{X})$. Let $\varphi(P(\delta, \alpha))=e_{(\delta, \alpha)} \otimes g_{(\delta, \alpha)}$. If Proposition 3.9 (1) holds, then $\frac{1}{2 \alpha}\left(\varphi(T+P(\delta, \alpha)) \varphi\left((x+\delta y) \otimes f_{(\delta, \alpha)}\right)+\varphi\left((x+\delta y) \otimes f_{(\delta, \alpha)}\right) \varphi(T+P(\delta, \alpha))\right)=$ 
$\frac{1}{2 \alpha}\left(\varphi(T+P(\delta, \alpha)) A(x+\delta y) \otimes C f_{(\delta, \alpha)}+A(x+\delta y) \otimes C f_{(\delta, \alpha)} \varphi(T+P(\delta, \alpha))\right) \in \mathcal{I}^{*}(\mathcal{X})$. This yields that $\varphi(T+P(\delta, \alpha)) A(x+\delta y)=\alpha A(x+\delta y)$ or $\varphi(T+P(\delta, \alpha))^{\prime} C f_{(\delta, \alpha)}=\alpha C f_{(\delta, \alpha)}$. When $\varphi(T+P(\delta, \alpha)) A(x+\delta y)=\alpha A(x+\delta y)$, we have that $h(A(x+\delta y)) z+g_{(\delta, \alpha)}(A(x+$ $\delta y)) e_{(\delta, \alpha)}=\alpha A(x+\delta y)$ for fixed $\delta \in \mathbb{C} \backslash\{0\}$ and any $\alpha \in \mathbb{C} \backslash\{0\}$. It follows that

$$
g_{\left(\delta, \alpha_{1}\right)}(A(x+\delta y)) e_{\left(\delta, \alpha_{1}\right)}-g_{\left(\delta, \alpha_{2}\right)}(A(x+\delta y)) e_{\left(\delta, \alpha_{2}\right)}=\left(\alpha_{1}-\alpha_{2}\right) A(x+\delta y)
$$

for fixed $\delta \in \mathbb{C}$ and any $\alpha_{1}, \alpha_{2} \in \mathbb{C} \backslash\{0\}$. Obviously,

$$
\left(1+\alpha_{2}-\alpha_{1}\right) g_{\left(\delta, \alpha_{1}\right)}(A(x+\delta y))=g_{\left(\delta, \alpha_{2}\right)}(A(x+\delta y)) g_{\left(\delta, \alpha_{1}\right)}\left(e_{\left(\delta, \alpha_{2}\right)}\right)
$$

for fixed $\delta \in \mathbb{C}$ and any $\alpha_{1}, \alpha_{2} \in \mathbb{C} \backslash\{0\}$. Hence, $g_{\left(\delta, \alpha_{2}\right)}(A(x+\delta y)) g_{\left(\delta, \alpha_{1}\right)}\left(e_{\left(\delta, \alpha_{2}\right)}\right)=0$ for fixed $\delta \in \mathbb{C}$ and any $\alpha_{1}, \alpha_{2} \in \mathbb{C} \backslash\{0\}$ with $1+\alpha_{2}=\alpha_{1}$. So, $g_{\left(\delta, \alpha_{2}\right)}(A(x+\delta y))=0$ or $g_{\left(\delta, \alpha_{1}\right)}\left(e_{\left(\delta, \alpha_{2}\right)}\right)=0$ for fixed $\delta \in \mathbb{C}$ and any $\alpha_{1}, \alpha_{2} \in \mathbb{C} \backslash\{0\}$ with $1+\alpha_{2}=\alpha_{1}$. According to Lemma 3.8, we know that $\varphi(P(\delta, \alpha)): \mathcal{X} \rightarrow[A(x+\delta y), A T(x+\delta y)]$ and so $g_{\left(\delta, \alpha_{1}\right)}\left(e_{\left(\delta, \alpha_{2}\right)}\right) \neq 0$. Hence, $g_{\left(\delta, \alpha_{2}\right)}(A(x+\delta y))=0$ for fixed $\delta \in \mathbb{C}$ and any $\alpha_{2} \in \mathbb{C} \backslash\{0\}$. With this observation, we know that $h(A(x+\delta y)) z=\alpha_{2} A(x+\delta y)$ for fixed $\delta \in \mathbb{C}$ and any $\alpha_{2} \in \mathbb{C} \backslash\{0\}$. This is a contradiction. When $\varphi(T+P(\delta, \alpha))^{\prime} f_{(\delta, \alpha)}=\alpha f_{(\delta, \alpha)}$, we get that $\alpha \in \sigma_{p}\left(z \otimes h+e_{(\delta, \alpha)} \otimes g_{(\delta, \alpha)}\right)$ for fixed $\delta \in \mathbb{C}$ and any $\alpha \in \mathbb{C} \backslash\{0\}$, where $\sigma_{p}\left(z \otimes h+e_{(\delta, \alpha)} \otimes g_{(\delta, \alpha)}\right)$ denotes that point spectrum of $z \otimes h+e_{(\delta, \alpha)} \otimes g_{(\delta, \alpha)}$. Note that $\mathcal{R}\left(z \otimes h+e_{(\delta, \alpha)} \otimes g_{(\delta, \alpha)}\right) \subseteq[z]+[A(x+\delta y), A T(x+\delta y)]$ for any $\alpha \in \mathbb{C}$. Here is a contradiction. If Proposition 3.9 (2) holds, then we get a contradiction again. Thus, $T$ is of rank-one.

The idea of the following proof comes from [9] and [13].

Proof of Theorem 1.2. The sufficiency is clear. Now, we prove the necessity and assume that $\varphi(I)=I$ by Lemma 3.3.

By Lemma 3.10, $\varphi$ maps the set of rank-one operators onto itself. This implies that the injective linear maps $A$ and $C$ mentioned in Proposition 3.9 are bijective. Suppose Proposition 3.9 (1) holds. By Lemma 3.4 and the linearity of $\varphi$, it can be shown that $C f(A x)=f(x)$ for all $x \in \mathcal{X}$ and $f \in \mathcal{X}^{\prime}$, which implies that $C$ is the adjoint of $A^{-1}$, and hence $C$ is bounded. Thus, $A^{-1}$ and $A$ are bounded too. Furthermore, for any $y \in \mathcal{X}$,

$$
\varphi(x \otimes f) y=(A x \otimes C f) y=(C f)(y) A x=f\left(A^{-1} y\right) A x=A(x \otimes f) A^{-1} y .
$$

Thus, $\varphi(T)=A T A^{-1}$ for any rank-one operator $T$. Moreover, every finite-rank operator can be written as a linear combination of finitely many rank-one operators. With the linearity of $\varphi$, we know that $\varphi(T)=A T A^{-1}$ for any finite-rank operator $T$. Replacing $\varphi$ by $A^{-1} \varphi A$, we may assume that $\varphi(T)=T$ for every finite-rank operator $T$. We next prove that $\varphi(T)=T$ for every non-scalar and infinite-rank operator $T \in \mathcal{B}(\mathcal{X})$. 
Let $T \in \mathcal{B}(\mathcal{X})$ be any non-scalar and infinite-rank operator. By the property of $\varphi$, we know that

$$
T R+R T \in \mathcal{I}(\mathcal{X}) \backslash\{0\} \Rightarrow \varphi(T) \varphi(R)+\varphi(R) \varphi(T)=\varphi(T) R+R \varphi(T) \in \mathcal{I}(\mathcal{X}) \backslash\{0\}
$$

for every $R \in \mathcal{F}_{1}(\mathcal{X})$. Suppose there is $x \in \mathcal{X}$ such that $\left(\varphi(A)+2 P_{0}\right) x$ and $\left(A+2 P_{0}\right) x$ are linearly independent for some rank-one idempotent $P_{0} \in \mathcal{I}^{*}(\mathcal{X})$ If $x$ and $\left(A+2 P_{0}\right) x$ are linearly independent, then there exists a rank-one idempotent $Q \in \mathcal{I}(\mathcal{X})$ such that $\left(A+2 P_{0}+2 Q\right) x=x$ by Lemma 2.4 . So, $\left(\varphi(A)+2 P_{0}+2 Q\right) x \neq\left(A+2 P_{0}+2 Q\right) x$ and there is $f \in \mathcal{X}^{\prime}$ such that $f\left(\left(\varphi(A)+2 P_{0}+2 Q\right) x\right)=0$ and $f\left(\left(A+2 P_{0}+2 Q\right) x\right)=1$. It follows that $\frac{1}{2}\left(\left(A+2 P_{0}+2 Q\right) x \otimes f+x \otimes f\left(A+2 P_{0}+2 Q\right)\right) \in \mathcal{I}^{*}(\mathcal{X})$ and then $\frac{1}{2}\left(\varphi\left(A+2 P_{0}+2 Q\right) x \otimes f+x \otimes f \varphi\left(A+2 P_{0}+2 Q\right)\right) \in \mathcal{I}^{*}(\mathcal{X})$. Hence, $f((\varphi(A)+$ $\left.\left.2 P_{0}+2 Q\right)^{2} x\right) x=2\left(\varphi(A)+2 P_{0}+2 Q\right) x$. Thus, $f\left(\left(\varphi(A)+2 P_{0}+2 Q\right)^{2} x\right)=0$ and so $\left(\varphi(A)+2 P_{0}+2 Q\right) x=0$. This is a contradiction. If $\left(A+2 P_{0}\right) x=\gamma x$ for some non-zero $\gamma \in \mathbb{C}$, then there is $g \in \mathcal{X}^{\prime}$ such that $g\left(\left(\varphi(A)+2 P_{0}\right) x\right)=0$ and $g((A+$ $\left.\left.2 P_{0}\right) x\right)=\gamma$. Hence, $\frac{1}{2 \gamma}\left(\left(A+2 P_{0}\right) x \otimes g+x \otimes g\left(A+2 P_{0}\right)\right) \in \mathcal{I}^{*}(\mathcal{X})$, which implies that $\frac{1}{2 \gamma}\left(\varphi\left(A+2 P_{0}\right) x \otimes g+x \otimes g \varphi\left(A+2 P_{0}\right)\right) \in \mathcal{I}^{*}(\mathcal{X})$. By direct calculation, we get that $g\left(\left(\varphi(A)+2 P_{0}\right)^{2} x\right) x=2 \gamma\left(\varphi(A)+2 P_{0}\right) x$ and so $f\left(\left(\varphi(A)+2 P_{0}+2 Q\right)^{2} x\right)=0$. Thus, $\left(\varphi(A)+2 P_{0}+2 Q\right) x=0$ and this is a contradiction. Thus, $\varphi(A)+2 P$ and $A+2 P$ are locally linearly dependent for any rank-one idempotent $P \in \mathcal{I}^{*}(\mathcal{X})$. By $[10$, Theorem 2.4], we know that there is $\eta(P) \in \mathbb{C}$ such that $\varphi(A)+2 P=\eta(P)(A+2 P)$ for any rank-one idempotent $P \in \mathcal{I}^{*}(\mathcal{X})$. Since $A+2 P$ is a non-scalar operator, we can find $x \in \mathcal{X}$ such that $x$ and $(A+2 P) x$ are linearly independent. Then there exists an idempotent operator $Q_{1} \in \mathcal{B}(\mathcal{X})$ of rank one such that $\left(A+2 P+2 Q_{1}\right) x=x$. There is $h \in \mathcal{X}^{\prime}$ such that $h(x)=1$. So, $\frac{1}{2}\left(\left(A+2 P+2 Q_{1}\right) x \otimes h+x \otimes h\left(A+2 P+2 Q_{1}\right)\right) \in \mathcal{I}^{*}(\mathcal{X})$. It follows that $\frac{1}{2}\left(\varphi\left(A+2 P+2 Q_{1}\right) x \otimes h+x \otimes h \varphi\left(A+2 P+2 Q_{1}\right)\right) \in \mathcal{I}^{*}(\mathcal{X})$. By direct calculation, we have that $\varphi\left(A+2 P+2 Q_{1}\right) x=x$ or $\varphi\left(A+2 P+2 Q_{1}\right)^{\prime} h=h$. Therefore, $\eta\left(P+Q_{1}\right)=1$ and then $\varphi(A)=A$.

Suppose Proposition 3.9 (2) holds. Then we have $(C x)(A f)=f(x)$ for all $x \in \mathcal{X}$ and $f \in \mathcal{X}^{\prime}$ by a similar argument. So, $C^{\prime}=A^{-1} K^{-1}$, where $K$ is the natural embedding of $X$ into $X^{\prime \prime}$. Thus, $A^{-1}$ is bounded and so as $\left(A^{-1}\right)^{\prime}$ and $C=\left(A^{-1}\right)^{\prime} K$. As $C$ and $\left(A^{-1}\right)^{\prime}$ are bijective, as so $K$ and hence $X$ is reflective. Now by a similar argument, $\varphi(T)=A T^{\prime} A^{-1}$ for every finite-rank operator $T$ and hence $\varphi(T)=A T^{\prime} A^{-1}$ for all every $T \in \mathcal{B}(\mathcal{X})$.

Acknowledgment. The author would like to thank the anonymous referees for their helpful comments and suggestions that greatly improved the original manuscript. 


\section{REFERENCES}

[1] M. Brešar and P. Šemrl. On locally linearly dependent operators and derivations. Trans. Amer. Math. Soc., 351:1257-1275, 1999.

[2] M. Dobovišek, B. Kuzma, G. Lešnjak, C.-K. Li, and T. Petek. Mappings that preserve pairs of operators with zero triple Jordan product. Linear Algebra Appl., 426:255-279, 2007.

[3] L. Fang and G.X. Ji. Linear maps preserving products of positive or Hermitian matrices. Linear Algebra Appl., 419:601-611, 2006.

[4] L. Fang, G.X. Ji, and Y.F. Pang. Maps preserving the idempotency of products of operators. Linear Algebra Appl., 426:40-52, 2007.

[5] J.C. Hou and J.L. Cui. Introduction to the Linear Maps on Operator Algebras. Science Press, Beijing, 2002.

[6] J.C Hou and Q.H. Di. Maps preserving numerical ranges of operator products. Proc. Amer. Math. Soc., 134:1435-1446, 2006.

[7] G.X. Ji and Y.L. Gao. Maps preserving operator pairs whose products are projections. Linear Algebra Appl., 433:1348-1364, 2010.

[8] C.-K. Li, P. Šemrl, and N.S. Sze. Maps preserving the nilpotency of products of operators. Linear Algebra Appl., 424:222-239, 2007.

[9] P. Šmrl. Similarity preserving linear maps. J. Operator Theory, 60(1):71-83, 2008.

[10] P. Šemrl. Non-linear commutativity preserving maps. Acta Sci. Math. (Szeged), 71:781-819, 2005.

[11] R. Meshulam and P. Šemrl. Locally linearly dependent operators. Pacific J. Math, 203:141-459, 2002.

[12] P. Šemrl. Linear maps that preserve the nilpotent operators. Acta Sci. Math. (Szeged), 61:523534, 1995.

[13] M.L. Wang, L. Fang, and G.X. Ji. Linear maps preserving idempotency of products or triple Jordan products of operatros. Linear Algebra Appl., 429:181-189, 2008. 